\title{
Epigenetic clock and methylation studies in the rhesus macaque
}

Steve Horvath ${ }^{1,2 \#}$, Joseph A. Zoller ${ }^{2 \#}$, Amin Haghani ${ }^{1}$, Anna J. Jasinska ${ }^{3}$, Ken Raj ${ }^{4}$, Charles E. Breeze ${ }^{5}$, Jason Ernst $^{6}$, Julie A. Mattison ${ }^{7}$

\section{Affiliations}

1 Department of Human Genetics, David Geffen School of Medicine, University of California, Los Angeles, Los Angeles, California, USA;

2 Department of Biostatistics, Fielding School of Public Health, University of California, Los Angeles, Los Angeles, California, USA;

3 Center for Neurobehavioral Genetics, Semel Institute for Neuroscience and Human Behavior, Department of Psychiatry and Biobehavioral Sciences, David Geffen School of Medicine, University of California, Los Angeles, Los Angeles, California, USA;

4 Radiation Effects Department, Centre for Radiation, Chemical and Environmental Hazards, Public Health England, Chilton, Didcot, UK;

5 Division of Cancer Epidemiology and Genetics, National Cancer Institute, National Institutes of Health, Department Health and Human Services, Bethesda, MD, USA;

6 Department of Biological Chemistry, University of California, Los Angeles, Los Angeles, CA, 90095, USA

7 Translational Gerontology Branch, National Institute on Aging Intramural Research Program, National Institutes of Health, USA;

\# Joint first authorship

Correspondence:

1) Steve Horvath E-mail: shorvath@mednet.ucla.edu Address: Gonda Building, 695 Charles Young Drive South, Los Angeles, CA 90095

2) Julie A. Mattison.Email: Julie.mattison@nih.gov

Emails:

shorvath@mednet.ucla.edu

jaz18@g.ucla.edu

ahaghani@g.ucla.edu

ankajasinska@gmail.com

ken.raj@phe.gov.uk

c.breeze@ucl.ac.uk

jason.ernst@ucla.edu

Julie.mattison@nih.gov

Key words: epigenetic clock, DNA methylation, rhesus monkey, nonhuman primate 


\begin{abstract}
Methylation levels at specific $\mathrm{CpG}$ positions in the genome have been used to develop accurate estimators of chronological age in humans, mice, and other species. Although epigenetic clocks are generally species-specific, the principles underpinning them appear to be conserved at least across the mammalian class. This is exemplified by the successful development of epigenetic clocks for mice and several other mammalian species. Here, we describe epigenetic clocks for the rhesus macaque (Macaca mulatta), the most widely used nonhuman primate in biological research. Using a custom methylation array (HorvathMammalMethylChip40), we profiled $n=281$ tissue samples (blood, skin, adipose, kidney, liver, lung, muscle, and cerebral cortex). From these data, we generated five epigenetic clocks for macaques. These clocks differ with regards to applicability to different tissue types (pan-tissue, blood, skin), species (macaque only or both humans and macaques), and measure of age (chronological age versus relative age). Additionally, the age-based human-macaque clock exhibits a high age correlation $(\mathrm{R}=0.89$ ) with the vervet monkey (Chlorocebus sabaeus), another Old World species. Four CpGs within the KLF14 promoter were consistently altered with age in four tissues (adipose, blood, cerebral cortex, skin). It is expected that the macaque clocks will reveal an epigenetic aging rate associated with a host of health conditions and thus lend themselves for identifying and validating anti-aging interventions.
\end{abstract}

\title{
INTRODUCTION
}

The rising costs of healthcare have fueled the growing need to address the root cause of most diseases and health conditions. Age is, without doubt, the strongest correlative factor across a wide range of pathologies. As such, investigations into the mechanisms and causes of aging, as well as interventions that might ameliorate its effects, hold great promise for improving health. To meet this end, animal models that closely recapitulate human aging are essential. Rhesus macaques (Macaca mulatta) are the most widely used nonhuman primate in biomedical research and share over 92\% DNA sequence homology with humans (1). They have an average lifespan in captivity of approximately 27 years, maximal lifespan of 42 years, and experience aging processes that are very similar to humans. With these features, the rhesus macaque presents as an excellent subject for the understanding of aging in humans and also other closely-related primate species $(2,3)$. Despite these attractive features, the employment of rhesus macaques in such research remains modest. This is due to both the prohibitive cost of maintaining a colony and the relatively long lifespan of these primates (4). These challenges, however, can be effectively addressed if accurate and robust biomarkers of biological age are established. Such biomarkers would change the experimental endpoint from longevity (measure of time from birth to death), to biological age (the measure of health and fitness). The application of biomarkers will greatly reduce the duration and cost of primate studies, while generating a much more meaningful understanding of why we age and provide the means to evaluate anti-aging interventions.

We report here, the development of DNA methylation-based biomarkers of age, known as epigenetic clocks. The development of such clocks was inspired by the vision that combining methylation levels of multiple CpGs that change with age would produce an accurate age estimator (reviewed in (5-7)). This notion was made possible by the technical advancement of array platforms that provide accurate quantitative measurements of methylation for thousands of specific $\mathrm{CpGs}$ in the genome and subsequently furthered the development of several human epigenetic clocks. Human and mouse pantissue DNA methylation (DNAm) age estimators exhibited important characteristics for aging studies, namely application to all sources of DNA (from sorted cells, tissues, and organs) and across the entire age spectrum (from prenatal tissue to centenarians) (5, 8-10). A substantial body of literature report that human epigenetic clocks capture many aspects of biological age (5). As such, the discrepancy between DNAm age and chronological age (termed as "epigenetic age acceleration") is predictive of all-cause mortality in humans even after adjusting for a variety of known risk factors (11-13). Several age related 
conditions are also associated with epigenetic age acceleration, including, but not limited to, cognitive and physical functioning (14), centenarian status $(13,15)$, Down syndrome (16), HIV infection (17), obesity (18).

Recently, mouse epigenetic clocks were successfully applied to evaluate and confirm benchmark longevity interventions such as calorie restriction and ablation of growth hormone receptor $(9,10,19$ 22). These observations strongly suggest that the capture of biological age by epigenetic clocks is not the preserve of human clocks but is a feature that applies several mammalian species. Since age-related DNA methylation change appears to be evolutionarily conserved, accurate age estimators, such as those developed for humans, may be applied across species. Although the human pan-tissue clock can indeed be applied to chimpanzee DNA methylation profiles, its performance with profiles of other animals decline as a result of evolutionary genome sequence divergence (8). Here we describe the development and performance of several epigenetic clocks for rhesus macaques, two of which are dual-species clocks that apply both to humans and rhesus macaques.

\section{RESULTS}

\section{DNA methylation data}

All rhesus macaque DNA methylation profiles were generated on a custom methylation array (HorvathMammalMethylChip40) that measures the methylation level of 36,000 CpGs with flanking DNA sequences that are conserved across the mammalian class. We obtained 281 DNA methylation profiles from 8 different tissues of rhesus macaque (Macaca mulatta) with ages that ranged from 1.8 years to 42 years (Table 1). An unsupervised hierarchical analysis clustered the methylation profiles by tissue type (Supplementary Figure 1). DNA methylation-based age estimators (epigenetic clocks) were developed using data from $n=281$ tissues, of which the most numerous were blood $(\mathrm{N}=199)$ and skin $(\mathrm{N}=51)$. Postmortem tissues (omental adipose, brain cortex, kidney, liver, lung, and skeletal muscle) were also available, but from fewer than 7 animals (Table 1). To generate dual-species epigenetic clocks that apply to both human and rhesus macaque, $n=850$ human tissue samples that were similarly profiled on HorvathMammalMethylChip40 were added to the rhesus macaque training set (Methods).

\section{Epigenetic clocks}

From these datasets, we generated five epigenetic clocks for macaques. These clocks differ with regards to applicability to different tissue types (pan-tissue, blood, skin), species (macaque only or both humans and macaques), and measure of age (chronological age versus relative age). As indicated by their names, pan-tissue clocks apply to all tissues, while the other clocks are developed for specific tissues/organs (blood, skin). The macaque pan-tissue clock was trained on all available tissues and applies only to rhesus macaques. The two human-macaque pan-tissue clocks, on the other hand, were derived from DNA methylation profiles from both species and are distinct from each other based on the unit of age that is employed. One estimates chronological age (in units of years), while the other estimates relative age, which is the ratio of chronological age to maximum lifespan, with values between 0 and 1 . This ratio allows alignment and biologically meaningful comparison between species with very different lifespan (rhesus macaque and human), which is not afforded by mere measurement of chronological age. The maximum recorded lifespans for rhesus macaques and humans are 42 years and 122.5 years, respectively, according to the updated version of the anAge data base (23), thus there is an approximate $3: 1$ age ratio. 
To arrive at unbiased estimates of the rhesus macaque pan-tissue clock, we carried out cross-validation analysis of the training data, followed by evaluation with an independent data set from another nonhuman primate species (vervet monkey). The cross-validation study reports unbiased estimates of the age correlation $\mathrm{R}$ (defined as Pearson correlation between the DNAm age estimate and chronological age) as well as the median absolute error.

The resulting macaque pan-tissue clock is highly accurate in age-estimation across tissues $(\mathrm{R}=0.95$, median absolute error 1.4 years, Figure 1A) and in individual types $(\mathrm{R}>=0.93$, Supplementary Figure 2C-I), except for adipose tissue ( $R=0.73$, Supplementary Figure 2B) for which only $n=5$ samples were available. The human-rhesus macaque clock for age is highly accurate when both species are analyzed together ( $\mathrm{R}=0.98$, Figure 1D), with a slight reduction when the analysis is restricted to rhesus macaque tissues $(\mathrm{R}=0.95$, Figure 1E).

Similarly, the human-rhesus macaque clock for relative age exhibits high correlation regardless of whether the analysis is done with samples from both species ( $R=0.97$, Figure 1F) or with only rhesus macaque samples ( $\mathrm{R}=0.95$, Figure 1G). The employment of relative age circumvents the inevitable unequal distribution of data at the opposite ends of the age range when chronological age of species with very different lifespans are measured using a single formula. A cross validation analysis reveals that both human-macaque clocks lead to high accuracy $(\mathrm{R}>=0.97)$ in human blood and skin samples (Figure 2).

\section{Cross-species performance of the rhesus macaque pan-tissue clock}

To determine the extent by which the rhesus macaque epigenetic clock can be applied to another primate, we used it to estimate the age of numerous tissues (blood, brain cortex, and liver) of the vervet monkey (Chlorocebus sabaeus), which is another Old World monkey separated 12.5 million years ago from the macaques. Despite this, we observed high correlations between the chronological age of vervets and their predicted age based on the macaque pan-tissue clock: $R=0.96$ in vervet blood, $R=0.92$ in vervet cortex, and $\mathrm{R}=0.98$ in vervet liver (Figure 3A-D). It is worth noting that the comparison of correlation coefficients between different tissues is not straightforward as these values are dependent on the age distribution of the samples that are evaluated (e.g. minimum and maximum age) and also on the sample size, albeit to a lesser extent. While the correlations are nevertheless impressively high, the level of concordance between chronological age and estimated age are less so, in particular with cortex, which exhibited an off set of 9 years (Figure 3B). Nevertheless, there is reasonably good concordance between chronological age of vervets and the estimated age of their blood (median error 1.7 years, Figure 3B) and liver (median error 3.5 years, Figure 3C) by the macaque pan-tissue clock.

\section{Epigenome-Wide Association Studies (EWAS) of chronological age in rhesus Macaque}

In total, 36,733 probes from HorvathMammalMethylChip40 could be mapped to specific loci in rhesus Macaque (Macaca mulatta.Mmul_10.100) genome. These loci are located proximal to 6154 genes. It is expected that findings resulting from the use of these clocks can be extrapolated to humans and other mammals since the mammalian array is designed to cover the most conserved regions across different mammalian genomes. To characterize the $\mathrm{CpGs}$ that change with macaque age (age-related CpGs) in different tissues, epigenome-wide association studies were carried out, which showed clear tissuespecificity of age-related CpGs (and their proximal genes) (Figure 4A). Hence, aging effects in one tissue do not appear to be reflected in another tissue (Supplementary Figure 3). This, however, may be owed to the limited sample size in non-blood tissue (Table 1). 
To identify CpGs whose methylation are most affected by age in all the tissues analyzed, DNAm changes were analyzed at a nominal $p$ value $<10^{-4}$. The top DNAm changes and their proximal genes in each tissue are as follows: adipose, $C H D 3$ promoter (Correlation test $\mathrm{Z}$ statistic $\mathrm{z}=-6$ ); blood, $V G F$ promoter ( $\mathrm{z}=16)$; cerebral cortex, PAX6 5' UTR $(\mathrm{z}=5)$; kidney, AGAP3 intron $(\mathrm{z}=-8.7)$; liver, ONECUT2 exon ( $\mathrm{z}=7.9)$; lung, distal intergenic region upstream of ZNF507 ( $\mathrm{z}=6.5)$, and GRIAl promoter ( $\mathrm{z}=-5.7)$; muscle, MNI intron ( $\mathrm{z}=-6.5)$; and skin, LHFPL4 intron ( $\mathrm{z}=11)$. Metaanalysis of these eight tissues, showed the top DNAm changes to include hypermethylation in $V G F$ promoter $(\mathrm{z}=14.8)$, four CpGs in $K L F 14$ promoter $(\mathrm{z}=12.7$ to 14.5$)$, SST promoter $(\mathrm{z}=12.9)$, and LHFPL4 exon $(\mathrm{z}=12.8)$ (Figure 4A). To identify CpGs that exhibited consistent age-associated methylation change across multiple (but not necessarily all) tissues, we generated an upset plot, which can be interpreted as a generalization of a Venn diagram. The upset plot analysis highlighted four CpGs in the KLF14 promoter as being age-related in at least 4 tissues (adipose, blood, cortex, and skin, Figure 4C). The KLF14 promoter controls expression of the KLF14 protein, which is itself a transcriptional factor that regulates the expression of TGFBII receptor.

Age-associated CpGs in different tissues were found to be distributed in genic and intergenic regions that can be defined relative to transcriptional start sites (Figure 4B). However, in tissues with sufficient sample numbers (blood and skin), CpGs located in promoters and 5'UTRs had a higher percentage of DNAm change than the background. Moreover, the DNAm changes in promoter and 5'UTR were mainly hypermethylation in all tissues. This result parallels prior observed patterns in DNAm aging in other species. We proceeded to identify putative transcriptional factors whose binding motifs were enriched for the top CpGs located in promoter or 5'UTR with DNAm changes, in either direction and in each tissue (Figure 4D). The top TF motifs were Zic1 and Zic2, which had 5 CpGs that become less methylated with age in muscle. These ZIC1 and ZIC2 transcription factors are particularly interesting because they regulate the expression of the $A P O E$ gene, which is associated with longevity and is the most commonly identified genetic risk factor of Alzheimer's disease (24). Thus, methylation change in this motif might underlie age-associated expression in this protein. For blood and lung, the top enriched motif is the TFAP2C (AP-2 gamma transcriptional factor) binding site that becomes increasingly hypomethylated with age. This motif also exhibited similar age-related changes in other mammalian species and is associated with genes that are involved in cell-cycle arrest, germ cell development, and implicated in several types of cancers $(25,26)$.

\section{Discussion}

Since the inception of the human pan-tissue epigenetic clock in 2013, the field has surged, and more epigenetic clocks have been developed for more applications incorporating different biological parameters that capture a wider range of health effects than mapped in earlier clocks. The human epigenetic clocks have many biomedical applications, including in human clinical trials where they can be used to assess the subjects' biological age in response to interventions $(5,27)$.

The utility of these human clocks prompted development of similar ones for other mammalian species. Clocks developed for mice are particularly important as they allow modelling of epigenetic age in an animal for which there is good biological understanding $(9,10,19-22)$. In addition to its impressive legacy in biological sciences, the advantage of a mouse model lies in no small part to its size, which facilitates its maintenance at an affordable cost, even at high numbers. Despite the many advantageous features, there is still a large gap in translating findings to primates. Hence, nonhuman primates play an indispensable role in preclinical investigations of potential interventions that might slow aging. As a case in point, both the National Institute on Aging and the University of Wisconsin have conducted longitudinal studies in rhesus macaques to determine whether the promising anti-aging intervention, 
caloric restriction, would also apply to primates and hence, more plausibly translate to human aging (28-30). Indeed, these studies have yielded valuable information about the role of diet composition, fasting timing, and overall intake on healthspan and lifespan(29-31). Despite their importance, such lifespan and healthspan studies in nonhuman primates can exceed the career span of the investigators and are costly. Therefore, the development of suitable biomarkers for biological age promises to greatly reduce the cost and time needed for carrying out such studies and can accelerate our ability to translate interventions.

As indicated, although the pan-tissue human clock can be directly applied to chimpanzees, which diverged from humans approximately 6.3 million years ago, it cannot be applied to any other nonhuman primates since they are more distantly related to humans. Thus, the development of specific epigenetic clocks for other nonhuman primate species is necessary. A critical step that obviates the species barrier was the development of a mammalian DNA methylation array (HorvathMammalMethylChip40) that profiles 36,000 CpGs with flanking DNA sequences that are conserved across multiple mammalian species. This allows DNA methylation profiling of virtually all mammalian species. The rhesus macaque DNA methylation profiles detailed here were derived from eight tissue types and represent the largest dataset to date of single-base resolution methylomes in highly conserved region across multiple tissues and ages.

This successful derivation of the multiple rhesus macaque epigenetic clocks attests, yet again, to the conservation of epigenetic aging mechanisms across the mammalian class. The macaque clock exhibits impressive age correlation with the vervet monkey clock, a species which diverged 12.5 million years ago. Moreover, the evolutionary conservation of epigenetic aging is further exemplified by demonstrating the feasibility of combining methylation profiles of humans and rhesus macaque. These species diverged 29 million years ago yet a single mathematical formula can be applied to generate human-rhesus macaque clocks. This single formula human-macaque clock is equally applicable to both species, and thereby demonstrates conservation of aging mechanisms, which alternatively could be deduced with the existence of multiple individual clocks for other mammals.

The significance of this unification under one formula has far reaching implications which extend beyond its utility in directly translating age-related findings in rhesus macaques to humans. With this tool, one can consider the root contributions to aging as it affirms the increasing evidence that aging is a coordinated biological process, harmonized throughout the body. This ushers in the possibility that when a regulator or coordinator of aging rate is identified, there is potential to modulate it through interventions. As this mechanism is conserved across species, interventions that successfully alter the epigenetic aging rate of rhesus macaques, as measured using the human-rhesus macaque clock, will likely exert similar effects in humans. If validated, this would be a milestone in aging research.

Although genome- and epigenome-wide analyses often yield a large number of potential target genes and pathways related to aging, it is not immediately obvious which ones are actually relevant. Yet, with repeated analyses of age-related $\mathrm{CpGs}$ in different species within the mammalian class, the relevant candidates can be identified. Here once again, the advantage of the HorvathMammalMethylChip40 comes to fore. As a case in point, analysis of datasets derived from this array revealed CpGs within the TFAP2 binding site were increasingly unmethylated with age across different mammalian species including rhesus macaque. Additionally, candidates such as Zic1 and Zic2, which did not feature in previously analyzed mammalian species, were uncovered and may indicate species-specific genes related to aging. Evolutionary selection and adaptation would predict a divergence in genes and pathways between species, and this is akin to other biological processes, such as cell cycle regulation, 
where a basic mechanism is conserved across species, but special additions, deletions, and modifications are identified in only a select species or group.

Just as there are species differences, age-related DNA methylation changes are tissue-specific. Sample size was a limitation of the current study, and thus we can draw only limited conclusions from our data presented here. This notwithstanding, it is interesting to note that CpGs within the KLF14 promoter were consistently altered with age in four tissues (adipose, blood, cerebral cortex, skin). KLF14 is a transcription factor that regulates the TGFBII receptor. This has potential physiological significance because the ligand of this receptor, TGFB, exerts diverse cellular effects including telomere regulation, unfolded protein response, autophagy, DNA repair, cellular senescence and stem cell aging. As a consequence, TGFB signaling is frequently involved in age-related pathologies such as cardiovascular disease, Alzheimer's disease, and osteoarthritis (32).

This is just one example from our extensive analysis of the rhesus epigenome that has broad tissue application and highlights the need for more in-depth empirical investigations to test and reveal the underlying mechanisms of epigenetic aging. Toward this end, the epigenetic clocks will play a pivotal role in uncovering potential candidates, monitoring aging rates, and testing putative aging interventions. The rhesus epigenetic clocks described here would play an inordinately important role in the translation of such interventions to humans.

\section{Materials and Methods}

\section{Materials}

\section{Rhesus macaque}

In total, we analyzed $\mathrm{N}=281$ rhesus macaque tissue samples from 8 different sources of DNA (Table 1). The rhesus monkeys have been housed continuously at the NIH Animal Center, Poolesville, MD. The animal center is fully accredited by the American Association for Accreditation of Laboratory Animal Care, and all procedures were approved by the Animal Care and Use Committee of the NIA Intramural Program. Monkeys were of a heterogenous genetic background, both Chinese and Indian origin.

Monkeys were housed individually in standard nonhuman primate caging on a $12 \mathrm{~h}$ light $/ 12 \mathrm{~h}$ dark cycle, room temperature $78+/-2$ degrees humidity at $60+/-20 \%$. All monkeys had extensive visual, auditory, and olfactory but limited tactile contact with monkeys housed in the same room. Monkeys received 2 meals per day at estimated ad libitum levels throughout the study. Water was always available ad libitum. Monkeys were monitored minimally 3 times daily by trained animal care staff.

\section{$\underline{\text { Sample Collection }}$}

Monkeys were fasted overnight, approximately 16-18 hours. Monkeys were anesthetized with either Ketamine, 7-10 mg/kg, IM or Telazol, 3-5 mg/kg, IM. Blood samples were obtained by venipuncture of the femoral vein using a vacutainer and EDTA tubes. Samples were immediately placed on dry ice and stored at -80 degrees. Skin samples were collected at the same time from an alcohol-wiped area of the back between the shoulder blades. Omental fat, kidney, liver, lung, skeletal muscle, and brain cortex were collected during necropsies scheduled for other study purposes. At that time, tissues were flash frozen in liquid nitrogen following collection and stored at -80 degrees. These tissues were selected for use based on having matching blood samples. None of the monkeys were sacrificed for this study.

\section{Vervet monkey animals}

The vervet monkey data are described in a companion paper (33). 


\section{Human tissue samples}

To build the human-rhesus macaque clock, we analyzed previously generated methylation data from $\mathrm{n}=850$ human tissue samples (adipose, blood, bone marrow, dermis, epidermis, heart, keratinocytes, fibroblasts, kidney, liver, lung, lymph node, muscle, pituitary, skin, spleen) from individuals whose ages ranged from 0 to 93 years. The tissue samples came from three sources: tissue and organ samples from the National NeuroAIDS Tissue Consortium (34), blood samples from the Cape Town Adolescent Antiretroviral Cohort study (35)., skin and other primary cells provided by Kenneth Raj (36). Ethics approval (IRB\#15-001454, IRB\#16-000471, IRB\#18-000315, IRB\#16-002028).

\section{DNA methylation profiling}

We generated DNA methylation data using the custom Illumina chip "HorvathMammalMethylChip40". By design, the mammalian methylation array facilitates epigenetic studies across mammalian species (including rhesus macaques and humans) due to its very high coverage (over thousand-fold) of highlyconserved $\mathrm{CpGs}$ in mammals. Toward this end, bioinformatic sequence analysis was employed to identify 36,000 highly conserved CpGs across 50 mammalian species (Arneson, Ernst, Horvath, in preparation). These $36 \mathrm{k}$ CpGs exhibit flanking sequences that are highly conserved across mammals. In addition, the custom array contains two thousand probes selected from human biomarker studies. Each probe is designed to cover a certain subset of species. The particular subset of species for each probe is provided in the chip manifest file and can be found at Gene Expression Omnibus (GEO) at NCBI as platform GPL28271. The SeSaMe normalization method was used to define beta values for each probe (37).

\section{Penalized Regression models}

Details on the clocks (CpGs, genome coordinates) and R software code are provided in the Supplement. Our pan-tissue clock for rhesus macaque is based on $71 \mathrm{CpGs}$ that are present on a custom chip (HorvathMammalMethylChip40). Our human-rhesus macaque epigenetic clock for chronological age is based on 508 CpGs. Another human-rhesus macaque epigenetic clock for relative age is based on 623 CpGs. We developed epigenetic clocks for rhesus macaques by regressing chronological age on the CpGs on the mammalian array. We used all tissues for the pan-tissue clock.

Penalized regression models were created with the R function "glmnet" (38). We investigated models produced by both "elastic net" regression (alpha $=0.5$ ). The optimal penalty parameters in all cases were determined automatically by using a 10 fold internal cross-validation (cv.glmnet) on the training set. By definition, the alpha value for the elastic net regression was set to 0.5 (midpoint between Ridge and Lasso type regression) and was not optimized for model performance. We performed a cross-validation scheme for arriving at unbiased (or at least less biased) estimates of the accuracy of the different DNAm based age estimators. One type consisted of leaving out a single sample (LOOCV) from the regression, predicting an age for that sample, and iterating over all samples.

For the cross-validation procedure, the penalized regression algorithm automatically selected a different sets of $\mathrm{CpGs}$ from the array for each fold. In case of $\mathrm{LOO}$ cross validation, the $\mathrm{CpG}$ selection was based on $\mathrm{n}-1$ observations. A critical step is the transformation of chronological age (the dependent variable). While no transformation was used for the pan-tissue clock for rhesus macaque, we did use a log linear transformation for the dual species clock of chronological age (Supplementary Methods).

Relative age estimation

To introduce biological meaning into age estimates of rhesus macaques and humans that have very different lifespan, as well as to overcome the inevitable skewing due to unequal distribution of data 
points from rhesus macaques and humans across age range, relative age estimation was made using the formula: Relative age $=$ Age/maxLifespan where the maximum lifespan for rhesus macaques and humans were set to 42 years and 122.5 years, respectively. The maximum lifespan for the two species was chosen from the updated version of the anAge data base (23).

\section{Epigenome wide association studies (EWAS) of age}

EWAS was performed in each tissue separately using the R function "standardScreeningNumericTrait" from the "WGCNA" R package (39). Next, the results were combined across tissues using Stouffer's meta-analysis method. Our epigenome wide association test studies of chronological age reveal that aging effects in one tissue are sometimes poorly conserved in another tissue.

\section{Transcription factor enrichment and chromatin states}

The FIMO (Find Individual Motif Occurrences) program scans a set of sequences for matches of known motifs, treating each motif independently (40). We ran TF motif (FIMO) scans of all probes on the HorvathMammalMethyl40 chip using motif models from TRANSFAC, UniPROBE, Taipale, Taipaledimer and JASPAR databases. A FIMO scan p-value of 1E-4 was chosen as cutoff (lower FIMO p-values reflect a higher probability for the local DNA sequence matching a given TF motif model). This cutoff implies that we find almost all TF motif matches that could possibly be associated with each site, resulting in an abundance of TF motif matches. We caution the reader that our hypergeometric test enrichment analysis did not adjust for CG content.

\section{URLs}

\section{Acknowledgements}

This study was supported by the Paul G. Allen Frontiers Group (PI SH). The rhesus macaque samples were contributed by the NIA NHP Core which is supported by the Intramural Research Program of the National Institute on Aging, NIH. Human tissue sample collection was supported by NIH funding through the NIMH and NINDS Institutes by the following grants: Manhattan HIV Brain Bank (MHBB): U24MH100931; Texas NeuroAIDS Research Center (TNRC): U24MH100930; National Neurological AIDS Bank (NNAB): U24MH100929; California NeuroAIDS Tissue Network (CNTN): U24MH100928 Data Coordinating Center (DCC): U24MH100925. Human blood samples were supported by R21MH107327. The contents are solely the responsibility of the authors and do not necessarily represent the official view of the NNTC or NIH.

\section{Conflict of Interest Statement}

$\mathrm{SH}$ is a founder of the non-profit Epigenetic Clock Development Foundation which plans to license several patents from his employer UC Regents. These patents list SH and JE as inventor. The other authors declare no conflicts of interest.

\section{Authors contributions}

The statistical analysis was carried out by JAZ, SH, ATL, AH, CB, JE. Rhesus tissues were provided by the NIA NHP Core and JAM. AJJ contributed vervet tissues. SH, KR, ATL drafted the article. JAM edited the manuscript. The study was conceived of by SH. 


\section{Figure legends}

\section{Leave-One-Out Analysis of All Final Epigenetic Clocks}
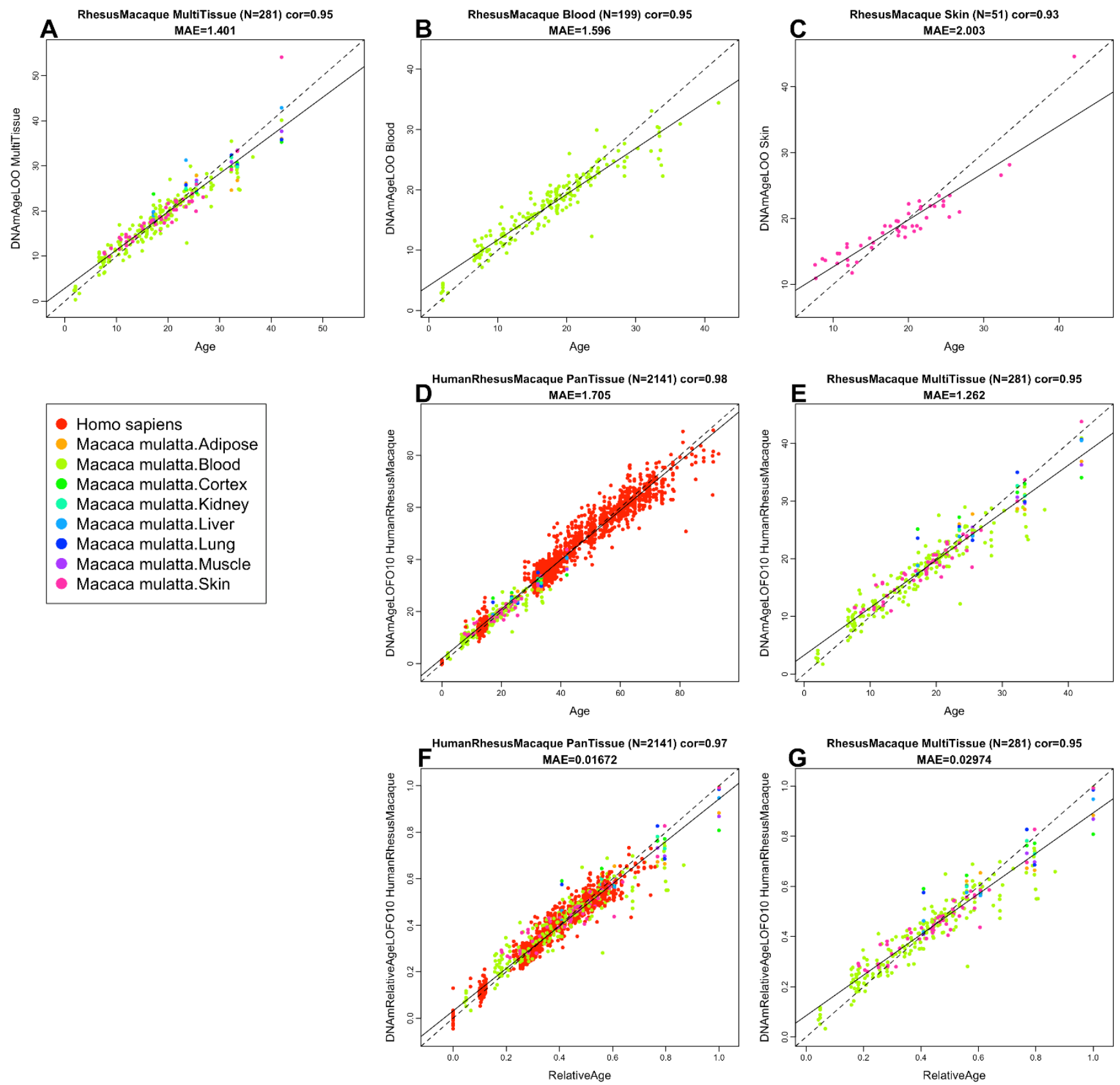

Figure 1: Cross-validation study of epigenetic clocks for rhesus macaques and humans. A-C)

Three epigenetic clocks that apply only to macaques. Leave-one-sample-out estimate of DNA methylation age (y-axis, in units of years) versus chronological age for A) all available macaque tissues, B) blood, C) skin. Ten -old cross validation analysis of the human-macaque monkey clocks for D,E) chronological age and F,G) relative age, respectively. D,F) Human samples are colored in red and macaque samples are colored by macaque tissue type, and analogous in E,G) but restricted to macaque samples (colored by macaque tissue type). Each panel reports the sample size (in parenthesis), correlation coefficient, median absolute error (MAE). 

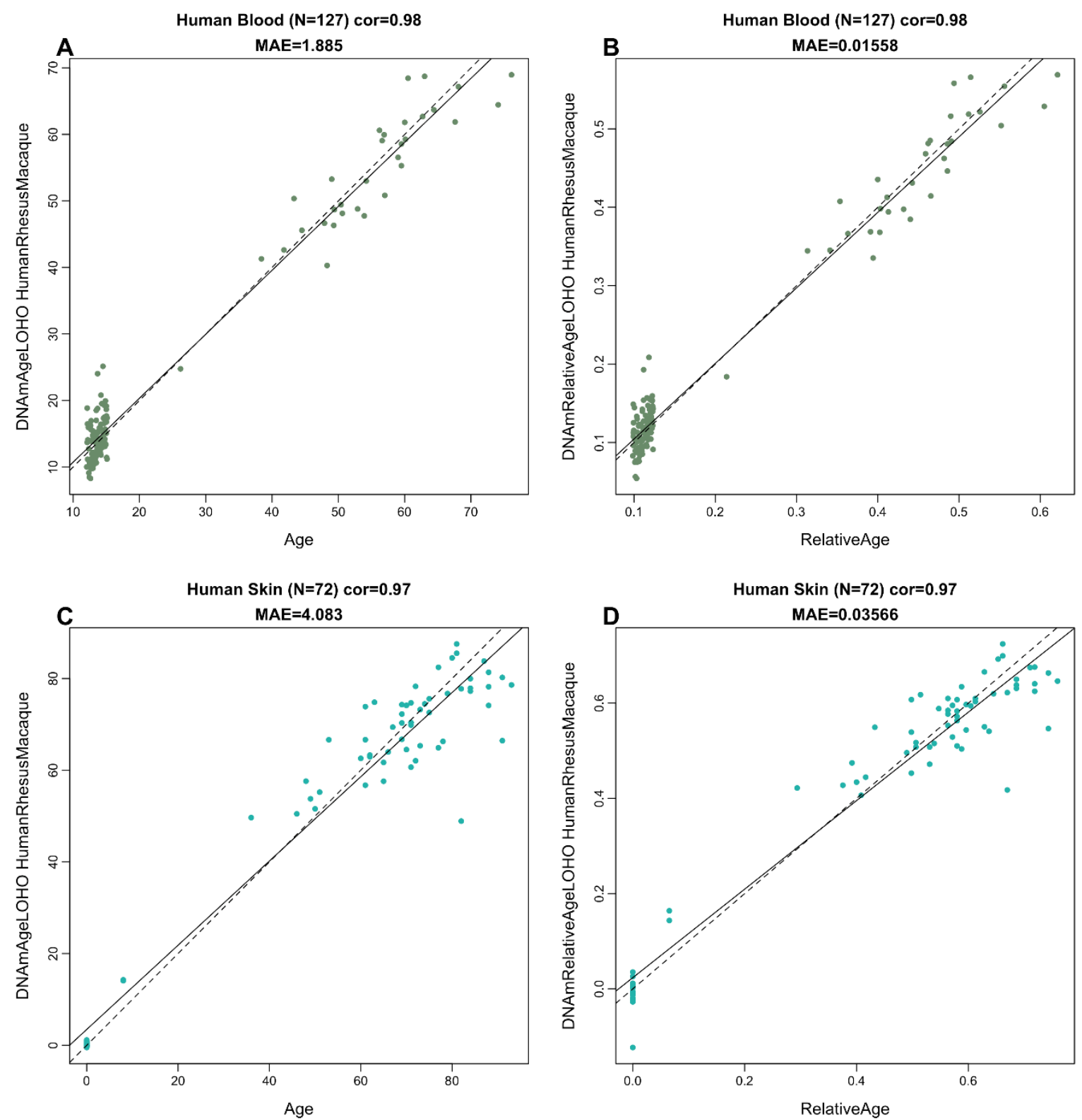

Figure 2. Human-macaque clocks applied to select human tissues. Leave-one-human sample-out (LOHO) cross fold cross validation estimates of the human-macaque clock for A,C) chronological age and C,D) relative age, respectively. A,B) Human blood samples. C,D) Human skin samples. Each panel reports the sample size (in parenthesis), correlation coefficient, median absolute error (MAE). 

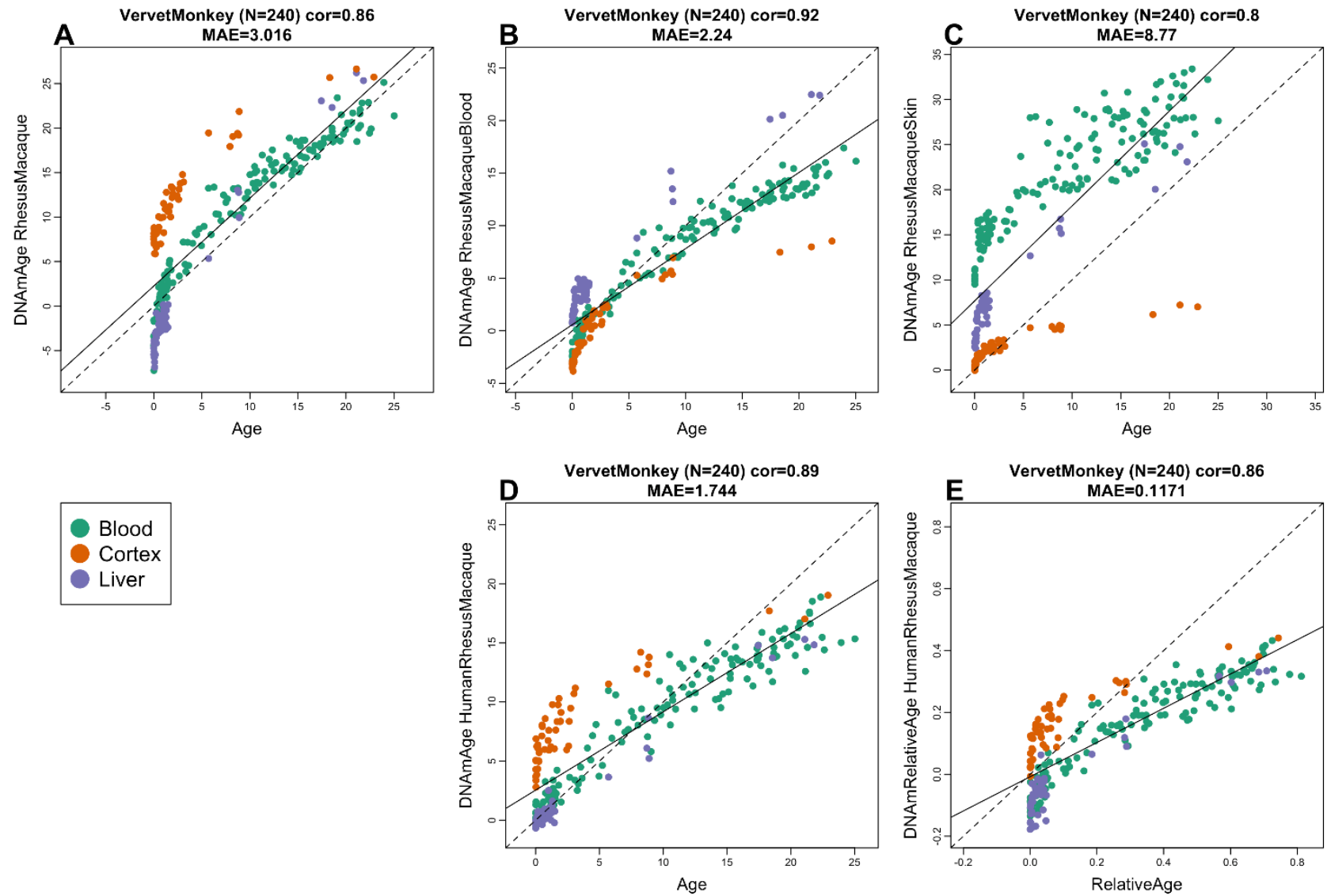

Figure 3. Macaque clocks applied to tissues from vervet monkey (Chlorocebus sabaeus). Each dot corresponds to a tissue sample from vervet monkeys. Each dot is colored by tissue type: blood (green), cerebral cortex (red), liver (purple). Chronological age of the vervet specimens (x-axis) versus the DNAm age estimate of the A) pan-tissue macaque clock, B) blood macaque clocks, C) skin macaque clock, D) human-macaque clock for chronological age, E) human-macaque clock for relative age. Each panel reports the sample size (in parenthesis), correlation coefficient, median absolute error (MAE). 
bioRxiv preprint doi: https://doi.org/10.1101/2020.09.21.307108; this version posted September 22, 2020. The copyright holder for this preprint (which was not certified by peer review) is the author/funder, who has granted bioRxiv a license to display the preprint in perpetuity. It is made available under aCC-BY-NC 4.0 International license.

A
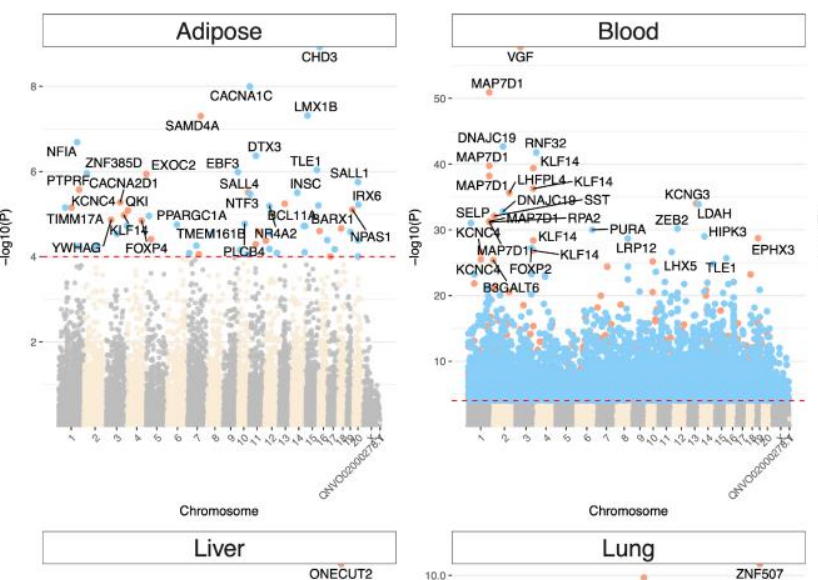

50. MAP7D1

$\begin{array}{lll}\text { DNAJC19 } & \text { RNF32 } \\ \text { 40- MAPTD1 } & \text { KLF14 }\end{array}$

MAPTD1 L LHFPLA KLF14

SELP KCNG3

MAP7D1 KLF14-PUAA ZEB2 LDAH LRP12 HIIPK3

BSGALT6
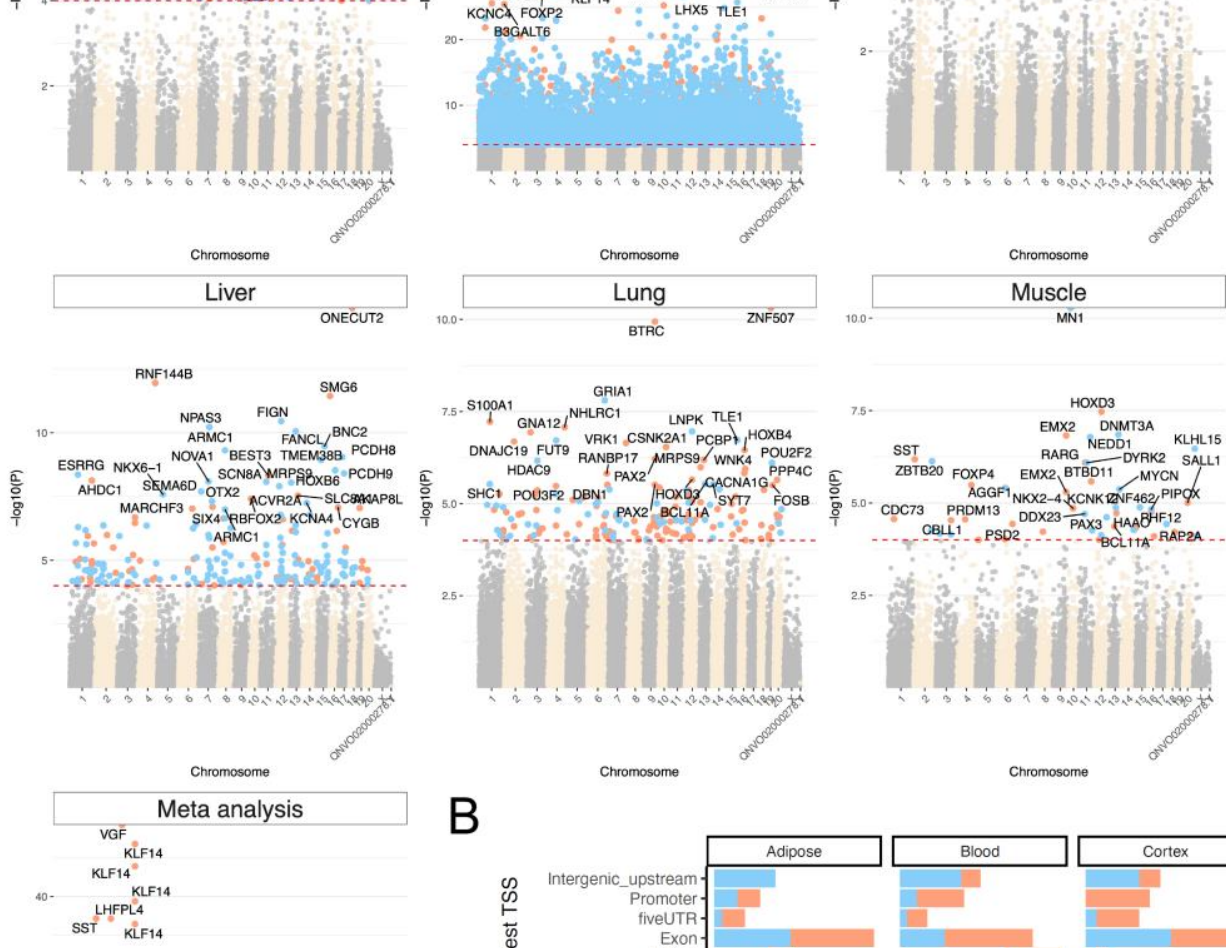

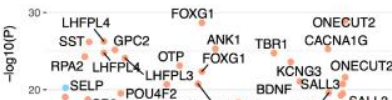
SPB POUAF2 ANKRD34C KCNH4 SAI3

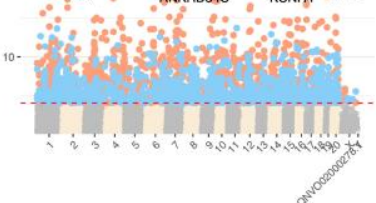

C

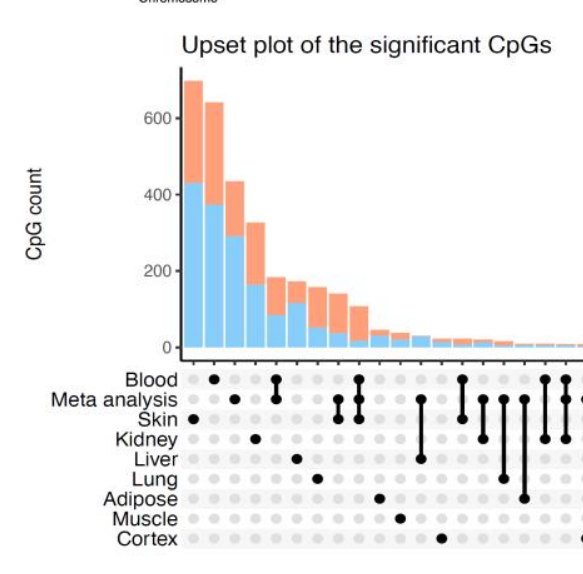

B
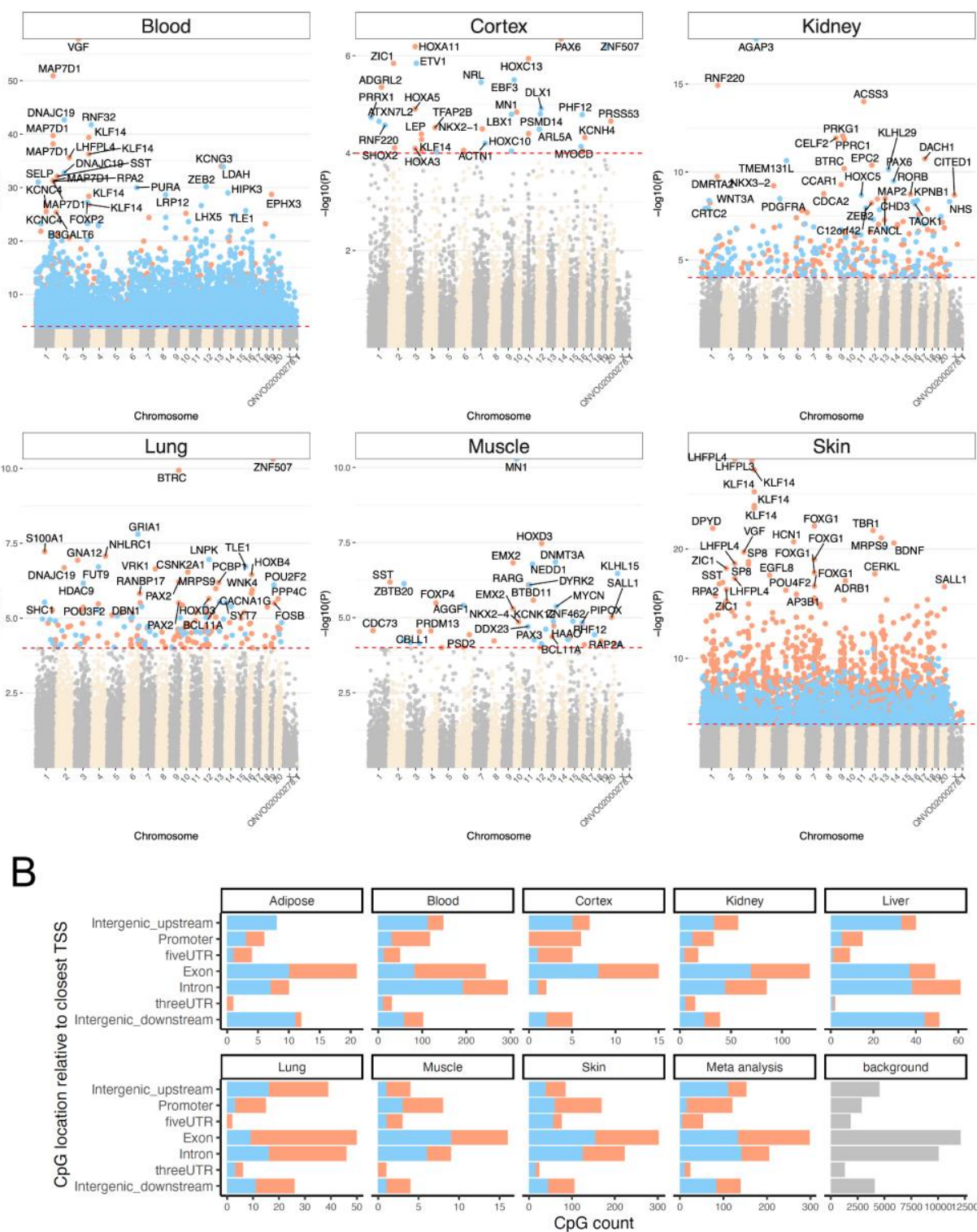

15. PNF22

ACss3 PRKG1 KLHL29
CELF2 PPRC1

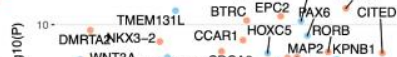

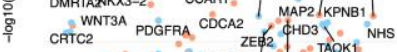
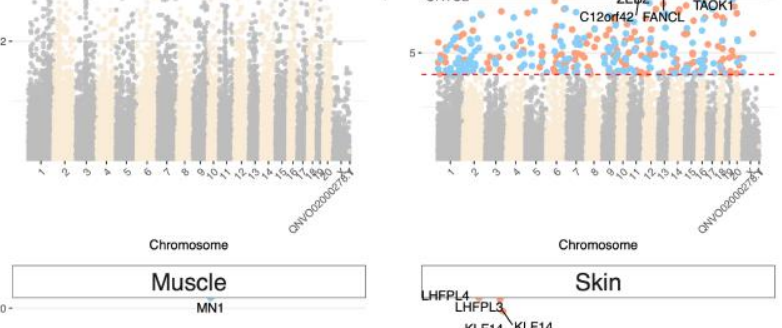
KLF14 KLF14
KLF14

KLF14

DPYD KLF14 FOXG1 TBR1

20. LHFPL4/ VPF HCN1 FOXG1 MRPS9 ${ }_{\text {BONF }}$

ZIC1 SPT EGFL8
SST FOXG1 CERKL E

RICA ILAFPL4 APB ADRB1

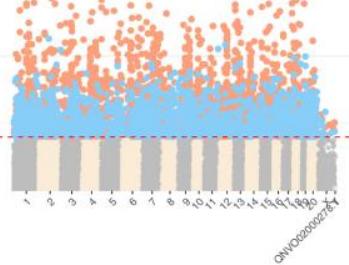

Chromosom
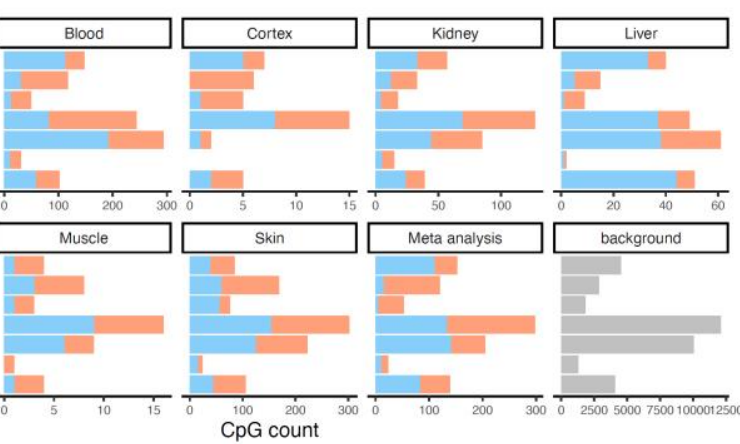

\section{Direction of association}

Hypermethylated Hypomethylated

D

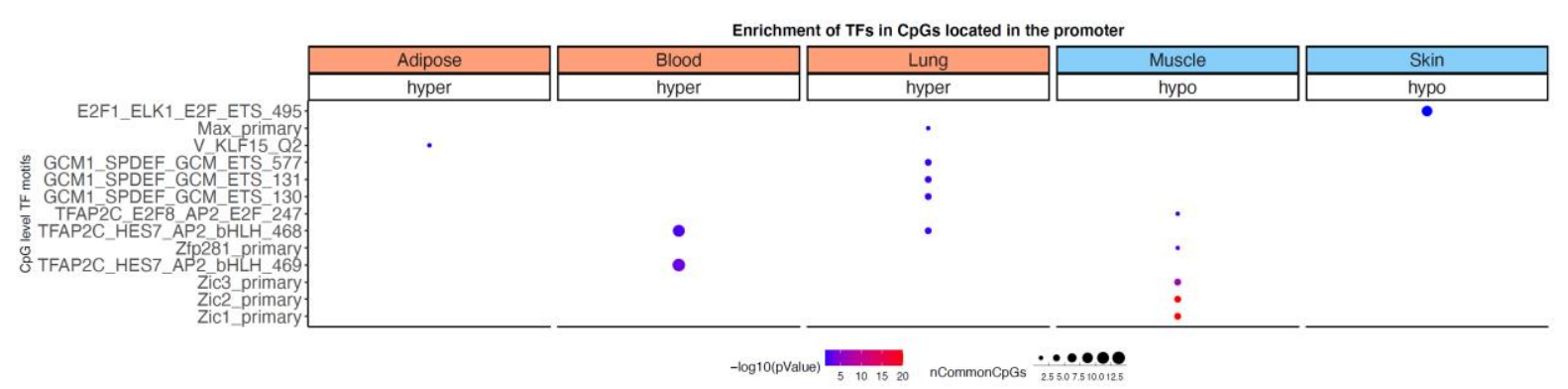


Figure 4. Epigenome-wide association (EWAS) of chronological age in adipose, blood, cerebral cortex, kidney, liver, lung, muscle, and skin of rhesus macaque. A) Manhattan plots of the EWAS of chronological age. The coordinates are estimated based on the alignment of Mammalian array probes to Mmul_10.100 genome assembly. The direction of associations with $\mathrm{p}<10^{-4}$ (red dotted line) is highlighted by red (hypermethylated) and blue (hypomethylated) colors. Top 30 CpGs was labeled by the neighboring genes. B) Location of top $\mathrm{CpGs}$ in each tissue relative to the closest transcriptional start site. Top CpGs were selected at $\mathrm{p}<10-4$ and further filtering based on $\mathrm{z}$ score of association with chronological age for up to 500 in a positive or negative direction. The number of selected CpGs: adipose, 62; blood, 1000; cerebral cortex, 40; kidney, 380; liver, 230; lung, 186; muscle, 47; skin, 1000; and meta-analysis, 1000. The grey color in the last panel represents the location of 36733 mammalian BeadChip array probes mapped to Mmul_10.100 genome. C) Upset plot representing the overlap of aging-associated $\mathrm{CpGs}$ based on meta-analysis or individual tissues. Neighboring genes of the overlapping CpGs were labeled in the figure. D) Transcriptional motif enrichment for the top CpGs in the promoter and 5 UTR of the neighboring genes. The enrichment was tested using a hypergeometric test (Methods).

\begin{tabular}{|c|c|c|c|c|c|}
\hline Tissue & N & No.Female & Mean.Age & Min.Age & Max.Age \\
\hline Adipose & 5 & 2 & 31.3 & 23.5 & 42 \\
\hline Blood & 199 & 71 & 17.2 & 1.79 & 42 \\
\hline Cortex & 6 & 3 & 29 & 17.2 & 42 \\
\hline Kidney & 4 & 1 & 28.7 & 23.5 & 33.4 \\
\hline Liver & 5 & 4 & 25.1 & 17.2 & 42 \\
\hline Lung & 6 & 3 & 29 & 17.2 & 42 \\
\hline Muscle & 5 & 2 & 30.1 & 17.2 & 42 \\
\hline Skin & 51 & 13 & 18.8 & 7.61 & 42 \\
\hline
\end{tabular}

Table 1. Description of biological materials from which DNA methylation profiles were derived. $\mathrm{N}=$ Total number of tissues. Number of females. Age: mean, minimum and maximum in units of years. 


\section{Supplementary Figures}

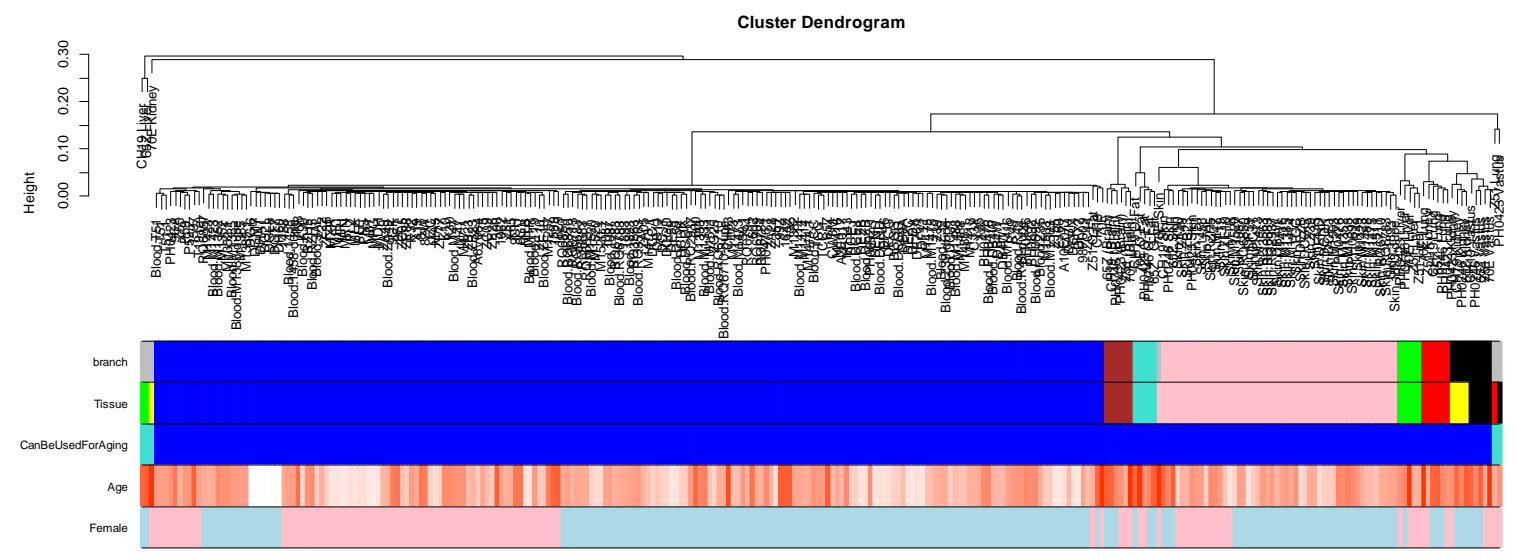

Supplementary Figure 1. Unsupervised hierarchical clustering of tissue samples.

Average linkage hierarchical clustering based on the interarray correlation coefficient (Pearson correlation). A height cut-off of 0.05 led to branch colors that largely correspond to Tissue type (second panel). A handful of arrays are severe outliers (left- and right most clusters) as indicated by the third color band (turquoise samples are severe outliers). These technical outliers probably result from insufficient amounts of DNA. These outlying samples were removed from the analysis. 

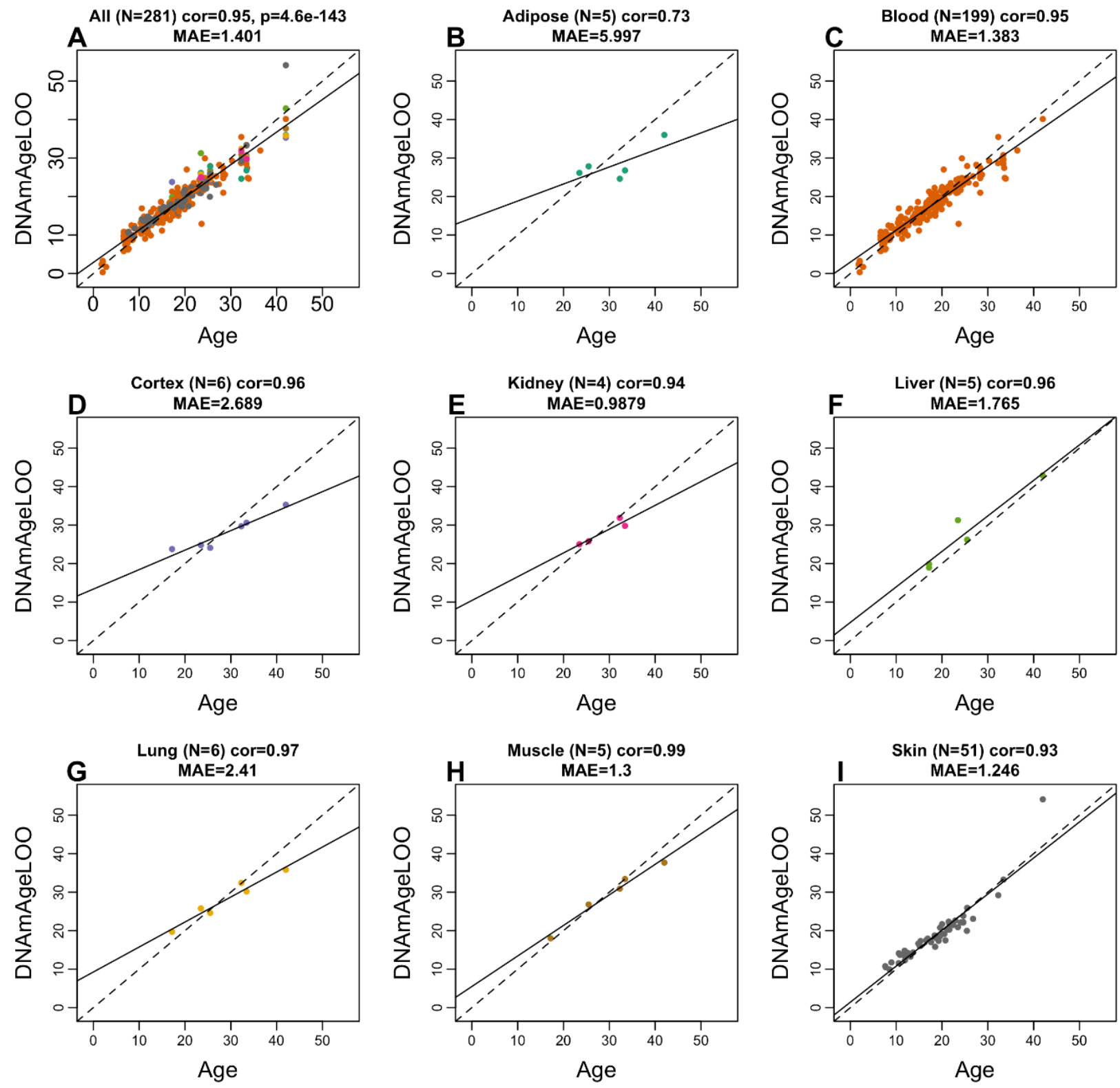

Supplementary Figure 2: Pan-tissue clock for rhesus macaque applied to different tissues. Each panel correlates the chronological age (x-axis) at the time of tissue collection with the leave-one-out (LOO) estimate of DNA methylation age. A) All tissues. Dots are colored by tissue type as indicated in the remaining panels. Results for B) adipose, C) blood, D) brain cortex, E) kidney, F) liver, G) lung, H) muscle, I) skin. Each panel reports the sample size (N), Pearson correlation, and the median absolute error, i.e. the median of the absolute difference between DNAmAgeLOO and chronological age. The dashed line is the diagonal $y=x$. The solid line corresponds to the least-squares regression line. 


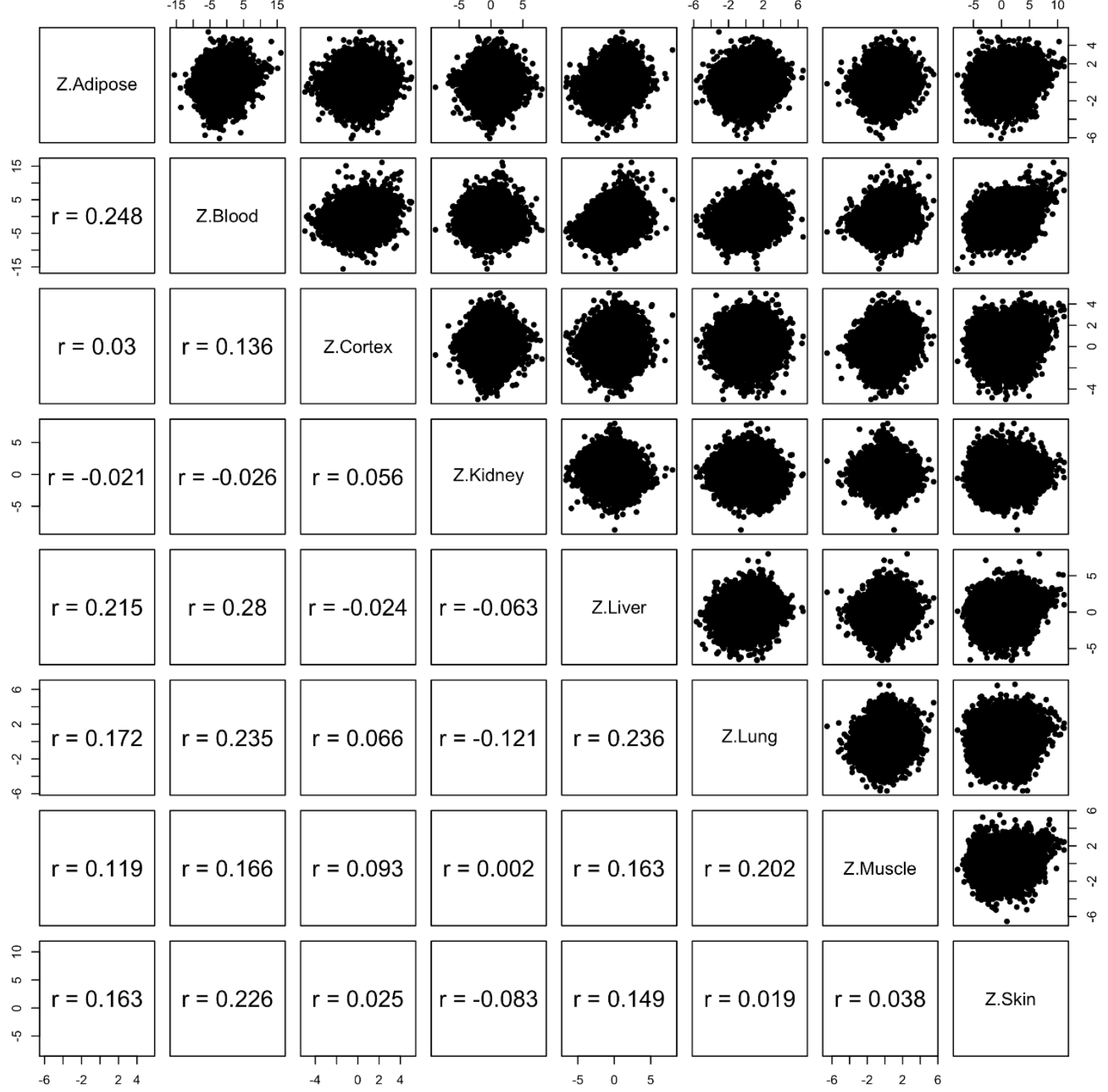

Supplementary Figure 3. EWAS was performed in each rat tissue separately using the R function "standardScreeningNumericTrait" from the "WGCNA" R package. Z statistics from correlation tests in different tissues. Upper panel report scatter plots for Z statistics in different tissues. Lower panels: corresponding Pearson correlation coefficients.

1. B. J. Bernacky, S. V. Gibson, M. E. Keeling, C. R. Abee, in Laboratory Animal Medicine (Second Edition), J. G. Fox, L. C. Anderson, F. M. Loew, F. W. Quimby, Eds. (Academic Press, Burlington, 2002), pp. 675-791.

2. G. S. Roth et al., Aging in rhesus monkeys: relevance to human health interventions. Science 305, 1423-1426 (2004).

3. R. A. Gibbs et al., Evolutionary and biomedical insights from the rhesus macaque genome. science 316, 222-234 (2007).

4. J. A. Mattison, K. L. Vaughan, An overview of nonhuman primates in aging research. Experimental gerontology 94, 41-45 (2017).

5. S. Horvath, K. Raj, DNA methylation-based biomarkers and the epigenetic clock theory of ageing. Nat Rev Genet, (2018). 
6. A. E. Field et al., DNA Methylation Clocks in Aging: Categories, Causes, and Consequences. Mol Cell 71, 882-895 (2018).

7. C. G. Bell et al., DNA methylation aging clocks: challenges and recommendations. Genome Biology 20, 249 (2019).

8. S. Horvath, DNA methylation age of human tissues and cell types. Genome Biol 14, R115 (2013).

9. M. J. Thompson et al., A multi-tissue full lifespan epigenetic clock for mice. Aging (Albany NY) 10, 2832-2854 (2018).

10. M. V. Meer, D. I. Podolskiy, A. Tyshkovskiy, V. N. Gladyshev, A whole lifespan mouse multi-tissue DNA methylation clock. eLife 7, e40675 (2018).

11. R. Marioni et al., DNA methylation age of blood predicts all-cause mortality in later life. Genome Biol. 16, 25 (2015).

12. B. H. Chen et al., DNA methylation-based measures of biological age: meta-analysis predicting time to death. Aging (Albany NY) 8, 1844-1865 (2016).

13. S. Horvath et al., Decreased epigenetic age of PBMCs from Italian semisupercentenarians and their offspring. Aging (Albany NY) 7, 1159-1170 (2015).

14. R. E. Marioni et al., The epigenetic clock is correlated with physical and cognitive fitness in the Lothian Birth Cohort 1936. Int J Epidemiol 44, 1388-1396 (2015).

15. S. Horvath et al., The cerebellum ages slowly according to the epigenetic clock. Aging (Albany NY) 7, 294-306 (2015).

16. S. Horvath et al., Accelerated epigenetic aging in Down syndrome. Aging Cell 14, 491-495 (2015).

17. S. Horvath, A. J. Levine, HIV-1 Infection Accelerates Age According to the Epigenetic Clock. J Infect Dis 212, 1563-1573 (2015).

18. S. Horvath et al., Obesity accelerates epigenetic aging of human liver. Proc Natl Acad Sci U S A 111, 15538-15543 (2014).

19. D. A. Petkovich et al., Using DNA Methylation Profiling to Evaluate Biological Age and Longevity Interventions. Cell Metab 25, 954-960 e956 (2017).

20. J. J. Cole et al., Diverse interventions that extend mouse lifespan suppress shared age-associated epigenetic changes at critical gene regulatory regions. Genome Biol 18, 58 (2017).

21. T. Wang et al., Epigenetic aging signatures in mice livers are slowed by dwarfism, calorie restriction and rapamycin treatment. Genome Biol 18, 57 (2017).

22. T. M. Stubbs et al., Multi-tissue DNA methylation age predictor in mouse. Genome Biol 18, 68 (2017).

23. J. P. de Magalhaes, J. Costa, G. M. Church, An analysis of the relationship between metabolism, developmental schedules, and longevity using phylogenetic independent contrasts. J Gerontol A Biol Sci Med Sci 62, 149-160 (2007).

24. E. Salero, R. Pérez-Sen, J. Aruga, C. Giménez, F. Zafra, Transcription factors Zic1 and Zic2 bind and transactivate the apolipoprotein $\mathrm{E}$ gene promoter. The Journal of biological chemistry 276, 1881-1888 (2001).

25. A. Bryant et al., miR-10a is aberrantly overexpressed in Nucleophosmin1 mutated acute myeloid leukaemia and its suppression induces cell death. Molecular Cancer 11, 8 (2012).

26. E. Penna et al., miR-214 coordinates melanoma progression by upregulating ALCAM through TFAP2 and miR-148b downmodulation. Cancer Res 73, 4098-4111 (2013).

27. G. M. Fahy et al., Reversal of epigenetic aging and immunosenescent trends in humans. Aging Cell 18, e13028 (2019).

28. A. V. Everitt, S. I. Rattan, D. G. Couteur, R. de Cabo, Calorie restriction, aging and longevity. (Springer Science \& Business Media, 2010).

29. R. J. Colman et al., Caloric restriction delays disease onset and mortality in rhesus monkeys. Science 325, 201-204 (2009).

30. R. J. Colman et al., Caloric restriction reduces age-related and all-cause mortality in rhesus monkeys. Nature communications 5, (2014). 
31. J. A. Mattison et al., Caloric restriction improves health and survival of rhesus monkeys. Nature Communications 8, 14063 (2017).

32. K. Tominaga, H. I. Suzuki, TGF- $\beta$ Signaling in Cellular Senescence and AgingRelated Pathology. Int J Mol Sci 20, (2019).

33. A. J. Jasinska et al., Epigenetic clock and methylation studies in vervet monkeys. bioRxiv, 2020.2009.2009.289801 (2020).

34. S. Morgello et al., The National NeuroAIDS Tissue Consortium: a new paradigm in brain banking with an emphasis on infectious disease. Neuropathol Appl Neurobiol 27, 326-335. (2001).

35. S. Horvath et al., Perinatally acquired HIV infection accelerates epigenetic aging in South African adolescents. AIDS (London, England) 32, 1465-1474 (2018).

36. S. Kabacik, S. Horvath, H. Cohen, K. Raj, Epigenetic ageing is distinct from senescence-mediated ageing and is not prevented by telomerase expression. Aging (Albany NY) 10, 2800-2815 (2018).

37. W. Zhou, T. J. Triche, Jr, P. W. Laird, H. Shen, SeSAMe: reducing artifactual detection of DNA methylation by Infinium BeadChips in genomic deletions. Nucleic Acids Research 46, e123-e123 (2018).

38. J. Friedman, T. Hastie, R. Tibshirani, Regularization Paths for Generalized Linear Models via Coordinate Descent. Journal of Statistical Software 33, 1-22 (2010).

39. P. Langfelder, S. Horvath, WGCNA: an R package for weighted correlation network analysis. BMC Bioinformatics 9, 559 (2008).

40. T. L. Bailey et al., MEME Suite: tools for motif discovery and searching. Nucleic Acids Research 37, W202-W208 (2009). 